\title{
Comportamento da mortalidade por câncer de mama nos municípios brasileiros e fatores associados
}

\author{
Maria Silvia de Azevedo Couto, ${ }^{1}$ Maximiliano Ribeiro Guerra, ${ }^{1}$ Vin ícius de \\ Azevedo Couto Firme ${ }^{3}$ e Maria Teresa Bustamante-Teixeira ${ }^{1}$
}

Como citar Couto MSA, Guerra MR, Firme VAC, Bustamante-Teixeira MT. Comportamento da mortalidade por câncer de mama nos municípios brasileiros e fatores associados. Rev Panam Salud Publica. 2017;41:e168. doi: 10.26633/RPSP.2017.168

RESUMO

Objetivo. Analisar o comportamento da mortalidade por câncer de mama nos municípios brasileiros e avaliar a influência de fatores socioeconômicos e demográficos sobre as taxas e mortalidade. Métodos. Foram calculadas taxas de mortalidade, padronizadas por faixa etária e corrigidas por causas mal definidas, centradas em 1990, 2000 e 2010. Posteriormente, foram estimados modelos de regressão, com dados em painel, que permitiram verificar o grau de associação entre os fatores de interesse e a taxa de mortalidade pela doença.

Resultados. Verificou-se uma tendência de crescimento da mortalidade no país. Contudo, os modelos indicaram que a mortalidade poderia ter diminuído (tendência negativa), principalmente no Sudeste e Sul, caso alguns fatores associados à doença (por exemplo, nível de renda, educação, longevidade, taxa de fecundidade, gastos em saúde, infraestrutura, entre outros) tivessem permanecido constantes durante o período considerado. Observou-se que a mortalidade por câncer de mama apresentou associação positiva/significativa com a longevidade e negativa/significativa com o nível de gastos públicos em saúde. A mortalidade foi maior nas regiões Sul e Sudeste, nos municípios com mais de 500000 habitantes e naqueles onde a população é inferior a 5000.

Conclusões. O crescimento da renda per capita, a elevação da expectativa de vida e a diminuição da taxa de fecundidade podem estar associados a elevadas taxas de mortalidade por câncer de mama e a uma tendência de crescimento na mortalidade por esse câncer nos municípios brasileiros.

Palavras-chave Neoplasias mamárias; mortalidade; epidemiologia; métodos e procedimentos estatísticos; Brasil.

Ao contrário do que ocorre em boa parte dos países desenvolvidos, que apresentam tendência de aumento da incidência e de redução da mortalidade por câncer de

\footnotetext{
Universidade Federal de Juiz de Fora (UFJF), Programa de Pós-Graduação em Saúde Coletiva, Juiz de Fora (MG), Brasil.

2 Inserm U900, Institut Curie, PSL Research University, Paris, França.

3 Universidade Federal de Juiz de Fora - Campus Governador Valadares (UFJF/GV), Governador Valadares (MG), Brasil. Correspondência: vinicius.firme@ufjf.edu.br.
}

mama (1-6), no Brasil a taxa de mortalidade por esse tipo de câncer cresceu nas últimas três décadas (7). Estudos indicam que a mortalidade por câncer de mama no país aumentou sistematicamente (8) em diferentes faixas etárias $(7,9,10)$. Contudo, esse crescimento não foi homogêneo, e algumas localidades apresentaram, inclusive, uma redução na mortalidade pela doença $(7,10)$.

A literatura reconhece que a taxa de mortalidade por câncer de mama varia conforme a região e o período analisados (10-12).
Essa heterogeneidade regional pode ser um reflexo de características geográficas, demográficas, socioeconômicas e culturais (2, 13-15). No Brasil, uma associação positiva foi relatada entre nível de renda e risco, incidência e mortalidade por câncer de mama $(13,16)$. Por outro lado, estudos sugerem que maiores níveis de escolaridade poderiam reduzir o risco associado ao câncer de mama (17), aumentar a sobrevida e facilitar o tratamento e detecção precoce da doença, contribuindo para a diminuição da

Este é um artigo de acesso aberto distribuído sob os termos da Licença Creative Commons Attribution-NonCommercial-NoDerivs 3.0 IGO, que permite o uso, distribuição e reprodução em qualquer meio, desde que o trabalho original seja devidamente citado. Não são permitidas modificações ou uso comercial dos artigos. Em qualquer reprodução do artigo, não deve haver nenhuma sugestão de que a OPAS ou o artigo avaliem qualquer organização ou produtos espeć́ficos. Não é permitido o uso do logotipo da OPAS. Este aviso deve ser preservado juntamente com o URL original do artigo 
mortalidade (18). Ademais, a escassez e a má distribuição de recursos físicos e humanos prejudicam o atendimento e o prognóstico da doença, principalmente em localidades de menor porte e afastadas das capitais. Esse fato pode sobrecarregar os centros oncológicos de referência, concentrados em localidades de grande porte (19, 20). Finalmente, os processos de industrialização também podem contribuir para aumentar o risco associado à doença pela exposição a fatores químicos, físicos e biológicos $(11,21)$.

Assim, o presente estudo teve por objetivo analisar o crescimento da mortalidade por câncer de mama observado em diferentes localidades do Brasil e verificar a influência dos fatores associados a essa neoplasia.

\section{MATERIAIS E MÉTODOS}

Trata-se de um estudo ecológico que utilizou os municípios brasileiros como unidades de análise e considerou as taxas médias de mortalidade por câncer de mama, padronizadas por idade, centradas nos anos de 1990 (média de 1987 a 1993), 2000 (média de 1997 a 2003) e 2010 (média de 2007 a 2013). Foram utilizados dados secundários não identificados, disponibilizados pelos órgãos responsáveis por sua coleta e divulgação, o que garante a confidencialidade.

\section{Fonte dos dados}

Foram utilizados os óbitos femininos por neoplasia maligna da mama ocorridos nos municípios brasileiros (tendo como referência a residência das falecidas) disponibilizados pelo Sistema de Informação sobre Mortalidade (SIM) do Departamento de Informática do SUS (DATASUS). Esses óbitos foram desagregados pelas faixas etárias da Organização Pan-Americana da Saúde (OPAS). Até 1995, o código 174 da Nona Revisão da Classificação Internacional de Doenças (CID-9) foi utilizado para identificar os óbitos. A partir de 1995, foi utilizado o código C50 da CID-10 (22).

Visando a minimizar o impacto da inexistência de registros de óbito por câncer de mama em alguns municípios, foram utilizadas as médias dos óbitos acumulados de 1987 a 1993 (CID-9), 1997 a 2003 (CID-10) e 2007 a 2013 (CID-10), centradas em 1990, 2000 e 2010, respectivamente. Tais médias foram utilizadas no cálculo da correção por causas mal definidas.

Como os óbitos por causas mal definidas podem comprometer a análise fidedigna das estatísticas de mortalidade (23), utilizou-se um procedimento (24) que consiste em calcular o percentual de correção por causas mal definidas (PCCMD) para cada município " $\mathrm{i}$ " no período " $\mathrm{t}$ " e o seu respectivo fator de correção, conforme as equações 1 e 2 :

$$
\text { PCCMD }_{i t}=\frac{\left(\text { totalobitosfem }_{i t}-\text { obitoscausasexternas }_{i t}\right)}{\left[\left(\text { totalobitosfem }_{i t}-\text { obitoscausasexternas }_{i t}\right)-\text { obitosmaldefinidos }_{i t}\right]}
$$

$$
F C_{i t}=1+\left(\frac{P C C M D_{i t}-1}{2}\right)
$$

Após o cálculo do PCCMD, multiplicase o fator de correção de cada município "i", no período " $\mathrm{t}$ " $\left(\mathrm{FC}_{\mathrm{it}}\right)$, pelo total de óbitos segundo faixa etária desse mesmo município no período " $\mathrm{t}$ ". Assim, obtêmse os óbitos corrigidos por causas mal definidas, tornando possível o cálculo das taxas específicas de mortalidade por câncer de mama $\left(\mathrm{MCM}_{\mathrm{it}}\right)$ por faixa etária e das taxas corrigidas por causas mal definidas. Portanto, as $\mathrm{MCM}_{\mathrm{it}}$ correspondem à média de óbitos corrigidos por causas mal definidas de cada município nos períodos citados, dividida pela população do município calculada pelo Instituto Brasileiro de Geografia e Estatística (IBGE) e disponibilizada pelo Instituto de Pesquisa Econômica Aplicada (IPEADATA) (25).

Para compor os óbitos por causas mal definidas (equações 1 e 2), utilizaram-se os óbitos femininos do capítulo XVI da CID-9 (sintomas, sinais e afecções mal definidas) para o período de 1987 a 1993. Para o período de 1997 a 2013, foram considerados os óbitos femininos do capítulo XVIII da CID-10 (sintomas, sinais e achados anormais de exames clínicos e de laboratório). Os óbitos totais, óbitos por causas mal definidas e óbitos por causas externas são disponibilizados pelo SIM através do DATASUS (22) e foram desagregados por faixa etária da OPAS.

As taxas de mortalidade, corrigidas por causas mal definidas, foram padronizadas por faixa etária via método direto, usando a população padrão mundial, estratificada por faixa etária (26). Considerando as variáveis mencionadas na introdução, determinou-se a equação 3:

$$
M C M_{i t}=f\left[\begin{array}{l}
I D H E_{i t}, I D H L_{i t}, I D H R_{i t}, I N D_{i t}, F E C_{i t}, M J F_{i t}, \\
\left(\frac{P R_{i t}}{P U_{i t}}\right), G P S_{i t}, N E S_{i t}, N P S_{i t}, D P M_{i}, D G_{i}, T E N D_{t}
\end{array}\right]
$$

senvolvimento socioeconômico de cada município. Os IDH municipais referentes a educação (IDHE), longevidade (IDHL) e renda (IDHR) foram obtidos do Atlas de Desenvolvimento Humano (27), assim como a taxa de fecundidade, o percentual de mulheres jovens (menores de 18 anos) com filhos e a proporção da população rural em relação à urbana.

Para a industrialização, utilizou-se o produto interno bruto (PIB) per capita municipal da indústria (valor adicionado a preços básicos), calculado pelo IBGE e convertido em valores constantes de 2000 com base no Índice Nacional de Preços ao Consumidor Amplo (IPCA) do IPEADATA. O gasto público per capita em saúde é fornecido pela Secretaria do Tesouro Nacional. Tanto os dados de industrialização quanto o gasto público per capita são disponibilizados pelo IPEADATA (25).

O número de estabelecimentos de saúde e de profissionais de saúde com ensino superior em cada município (por 100000 habitantes) estão disponíveis no TabNet, módulo rede assistencial do DATASUS (22). Para 1991 e para 2000, foram utilizados os dados da Pesquisa Assistência Médico-Sanitária de 1992 e 1999, respectivamente. Os dados de 2010 estão no Cadastro Nacional de Estabelecimentos de Saúde.

Foram criadas dummies geográficas para os municípios das regiões Sul, Centro-Oeste, Nordeste e Norte. Por esse procedimento, todos os municípios pertencentes à região em análise recebem valor de 1 , enquanto os municípios das outras regiões recebem o valor de zero - de forma que seja possível captar o efeito de pertencer a 
uma região específica, permitindo verificar se a taxa de mortalidade é diferente entre regiões. O Sudeste foi tratado como referência. Também foram criadas dummies de porte populacional. Nesse último caso, os municípios " $\mathrm{i}$ " foram subdivididos de acordo com porte em $\mathrm{i}<5 ; 5 \leq \mathrm{i}<10 ; 10 \leq \mathrm{i}<20$; $20 \leq \mathrm{i}<50 ; 50 \leq \mathrm{i}<100 ; 100 \leq \mathrm{i}<500 ; 500 \leq \mathrm{i}<1000$; e i $\geq 1000$, onde os valores representam milhares de habitantes (os municípios com menos de 5000 habitantes, $i<5$, foram considerados como referência).

As categorias de referência servem como base de comparação. Assim, a constante estimada refletirá a taxa de mortalidade dos municípios com menos de 5000 habitantes (referência) pertencentes à região Sudeste (referência).

Devido ao período e à unidade de análise utilizada (municípios), fatores como idade na menarca e na menopausa, tipo de alimentação e percentual de fumantes não puderam ser incluídos nos modelos. Optou-se por não utilizar a cor da pele devido ao elevado percentual de dados ausentes e aos frequentes erros de classificação associados a essa variável (28). Ademais, como o DATASUS (22) passou a divulgar o número de mamógrafos por município apenas em 1999, essa variável também foi desconsiderada.

A correlação excessiva entre as variáveis explicativas (equação 3) poderia gerar multicolinearidade, comprometendo as estimações. Contudo, o teste de fator de inflação da variância (vector inflation factor, VIF), que mede quanto cada variável explicativa " $k$ " está associada às demais, não indicou a presença desse problema. Formalmente, tem-se: $V I F=1 /\left(1-R_{k}^{2}\right)$, onde $R_{k}^{2}$ representa o $\mathrm{R}^{2}$ tradicional oriundo da estimação da variável " $\mathrm{k}$ " contra as demais explicativas (29).

\section{Análise dos dados}

Os dados foram agrupados em um painel balanceado, onde uma mesma unidade de corte transversal (neste caso, os municípios brasileiros) foi analisada ao longo do tempo, isto é, 1990, 2000 e 2010. Esta opção permitiu considerar mais observações (melhorando as propriedades assintóticas dos estimadores-estatísticas $t$ e F mais robustas), controlar efeitos não observados (" $c$ ") constantes no tempo (por exemplo, cultura, clima e relevo) e realizar análises dinâmicas (por exemplo, análise de tendência) (30).

No contexto de dados de painel, geralmente estima-se um modelo do tipo pooled ordinary least squares (POLS) usando o teste de Breusch-Pagan para verificar se existe algum ci afetando os resultados (31). Nesse caso, a hipótese nula é: $H_{0}:\left(\sigma_{c}^{2}\right)=0$, onde $\sigma_{c}^{2}$ representa a variância do efeito não observado "c". Caso a hipótese nula prevaleça, o modelo POLS é o mais indicado. Caso a hipótese nula não se confirme, estimam-se modelos de efeitos fixos e aleatórios, usando o teste de Hausman (32) para definir o mais adequado. Esse teste verifica se as variáveis explicativas $\left(X_{i t}\right)$ estão correlacionadas com $c_{i}$, sendo $H_{0}: E\left[\left(c_{\mathrm{i}} \mid X_{i t}\right)=0\right]$. Se $H_{0}$ for verdadeiro, ambos os modelos serão consistentes, porém o modelo aleatório será mais eficiente. Do contrário, o teste de Breusch-Pagan se torna desnecessário e somente o modelo de efeitos fixos será consistente (31).

É importante notar que somente o estimador de efeitos fixos é capaz de eliminar o viés causado por $c_{i}$. Para tanto, utilizam-se os desvios de cada variável $x_{t}$ em relação a sua média $\bar{x}$, ou seja: $\ddot{x}_{t}=x_{t}-\bar{x}(31)$. Exemplo: considere que "Sul" seja utilizada como dummy para refletir a cultura dessa região. Como um município " $\mathrm{i}$ " da região não mudará sua localização nos anos analisados, a dummy será igual a 1 em 1990, 2000 e 2010, e também terá média igual a 1. Logo, a dummy (e qualquer outro $c_{j}$ ) acaba eliminada do modelo, pois: $\ddot{x}_{t}=\left(x_{t}-\bar{x}\right)=(1-1)=0$.

A variável utilizada para captar a tendência associada à mortalidade por câncer de mama foi função exclusiva do período considerado (tendência determinística), ou seja: $M C M_{i t}=T_{e n d}+\varepsilon_{t}$, onde $\mathrm{MCM}_{\mathrm{it}}$ é a mortalidade de cada município " $\mathrm{i}$ " no período " $\mathrm{t}$ "; Tend $\mathrm{t}_{\mathrm{t}}$ representa a tendência, com valores iguais a 1 (em 1990), 2 (em 2000) e 3 (em 2010); e $\varepsilon_{t}$ é um erro aleatório bem comportado. Assim, foi possível avaliar a evolução da mortalidade por câncer de mama (MCM), em valores absolutos, para cada período analisado (29).

A inclusão/exclusão de variáveis se baseou no critério de informação de Akaike (AIC) e no critério de informação Bayesiano (BIC). Ambos se valem do valor da função de máxima verossimilhança como medida de ajustamento e penalizam o excesso de variáveis incluídas. Quanto menor o valor de AIC e BIC, melhor o ajustamento (29). A estratégia empírica consistiu em incluir "grupos" de variáveis (dummies de região e porte municipal, variável de tendência e variáveis contínuas, como renda, longevidade, educação, industrialização, fecundidade, número de mulheres jovens grávidas, população urbana/rural, gasto público em saúde, número de profissionais e estabelecimentos de saúde), a fim de verificar como a inclusão de um novo grupo afetaria os resultados previamente obtidos.

O software STATA foi usado para estimativas e resultados estatísticos apresentados nesta pesquisa.

\section{RESULTADOS}

A análise descritiva das variáveis revelou que a taxa de MCM dos municípios brasileiros, padronizada por faixa etária e corrigida por causas mal definidas, foi, em média, 9,66/100 000 no período analisado. No mesmo período, verificou-se aumento do IDHR, IDHL e IDHE. Já a produção industrial per capita municipal foi equivalente a 1254,14 reais $(685,54$ dólares) (tabela 1).

A taxa de fecundidade diminuiu de 3,20, em 1990, para 1,99 em 2010. A média do período foi de 2,59 filhos por mulher. Já o percentual de mulheres jovens com filhos cresceu. Em média, aproximadamente $2,8 \%$ das mulheres com menos de 18 anos possuíam filhos. O coeficiente (menor que 1) associado à razão população rural/população urbana indicou que a maior parte das pessoas residia em áreas urbanas.

No ano 2000, o gasto público em saúde avaliado em reais foi equivalente a $R \$$ 101,30 per capita (US\$ 55,37). O número de profissionais de saúde foi de 337,39/100000 habitantes. Por sua vez, o número de estabelecimentos de saúde foi de 45/100 000 habitantes. Todas as três variáveis aumentaram no período (tabela 1 ).

Como mostra a tabela 1, 39\% dos 2311 municípios considerados pertencem ao Sudeste e aproximadamente $29 \%$ tinham entre 20 e 50000 habitantes. Os valores da variável de tendência são iguais a 1 (em 1990), 2 (em 2000) e 3 (em 2010). Para a tendência associada às regiões, os valores mencionados são atribuídos apenas aos municípios da região analisada (para os demais, atribui-se o valor zero).

Os resultados da tabela 2 indicam que houve uma tendência de crescimento da mortalidade por câncer de mama no Brasil (coeficientes positivos). Verificou-se, ainda, uma diferença regional significativa associada à mortalidade. Segundo o modelo "a", a mortalidade foi maior na região Sul (mais rica) e menor na região Norte (mais pobre). Como a região Sudeste foi a referência, sua taxa é igual à constante $(9,94)$ mais a tendência $(0,22)$. 
TABELA 1. Análise descritiva das variáveis associadas ao câncer de mama em municípios brasileiros, 1990 a 2010

\begin{tabular}{|c|c|c|c|c|}
\hline$\overline{\text { Variável }^{a}}$ & Média & Desvio padrão & Mínimo & Máximo \\
\hline \multicolumn{5}{|c|}{ Corte transversal: 1990 (observações: 2 311) } \\
\hline MCM & 9,53 & 5,88 & 0,47 & 50,74 \\
\hline IDHR & 0,57 & 0,09 & 0,32 & 0,80 \\
\hline IDHE & 0,23 & 0,09 & 0,02 & 0,56 \\
\hline Industrialização $0^{\mathrm{b}, \mathrm{c}}$ & 874,06 & 2356,17 & 0,01 & 74216,37 \\
\hline Fecundidade & 3,20 & 0,90 & 1,76 & 8,29 \\
\hline $\mathrm{GPS}^{\mathrm{c}, \mathrm{d}}$ & 25,02 & 23,37 & 0,00 & 252,15 \\
\hline NPS & 172,43 & 176,19 & 0,00 & 6434,56 \\
\hline NES & 33,79 & 25,14 & 0,00 & 215,78 \\
\hline \multicolumn{5}{|l|}{ Corte transversal: 2000} \\
\hline MCM & 9,47 & 5,45 & 0,43 & 47,32 \\
\hline IDHR & 0,62 & 0,08 & 0,37 & 0,86 \\
\hline Fecundidade & 2,58 & 0,52 & 1,56 & 6,31 \\
\hline Mulheres jovens com filhos & 3,36 & 1,74 & 0,00 & 16,04 \\
\hline População rural/população urbana & 0,63 & 1,51 & 0,00 & 63,77 \\
\hline GPS $^{\mathrm{c}, \mathrm{d}} \mathrm{s}^{2}$ & 78,91 & 108,24 & 0,00 & 4702,09 \\
\hline NPS & 260,16 & 307,12 & 0,00 & 8351,03 \\
\hline NES & 39,00 & 23,38 & 0,00 & 214,82 \\
\hline \multicolumn{5}{|l|}{ Corte transversal:2010 } \\
\hline MCM & 9,98 & 4,53 & 0,84 & 43,90 \\
\hline IDHR & 0,68 & 0,07 & 0,50 & 0,89 \\
\hline IDHL & 0,82 & 0,04 & 0,68 & 0,89 \\
\hline IDHE & 0,60 & 0,08 & 0,33 & 0,81 \\
\hline Industrialização $0^{\mathrm{b}, \mathrm{c}}$ & 1756,07 & 3765,65 & 116,91 & 70961,45 \\
\hline IDHR & 0,62 & 0,09 & 0,32 & 0,89 \\
\hline IDHL & 0,75 & 0,08 & 0,44 & 0,89 \\
\hline IDHE & 0,42 & 0,18 & 0,02 & 0,81 \\
\hline Industrialização $0^{\mathrm{b}, \mathrm{c}}$ & 1254,14 & 2870,97 & 0,01 & 74216,37 \\
\hline Fecundidade & 2,59 & 0,81 & 1,21 & 8,29 \\
\hline Mulheres jovens com filhos & 2,80 & 1,62 & 0,00 & 16,04 \\
\hline População rural/população urbana & 0,67 & 1,31 & 0,00 & 63,77 \\
\hline GPS $^{c, d}$ & 101,30 & 113,04 & 0,00 & 4702,09 \\
\hline NPS & 337,39 & 328,91 & 0,00 & 8351,03 \\
\hline NES & 45,19 & 28,35 & 0,00 & 303,48 \\
\hline \multicolumn{5}{|l|}{ Dummies geográficas ${ }^{e, f}$} \\
\hline Sudeste & 0,39 & NA & 0 & 1 \\
\hline Sul & 0,26 & NA & 0 & 1 \\
\hline Centro-Oeste & 0,07 & NA & 0 & 1 \\
\hline Norte & 0,03 & NA & 0 & 1 \\
\hline Nordeste & 0,25 & NA & 0 & $1 \mathrm{v}$ \\
\hline Total & 1,00 & & & \\
\hline \multicolumn{5}{|l|}{ Dummies de porte municipale,f } \\
\hline Até 5000 & 0,06 & NA & 0 & 1 \\
\hline 5 a 10000 & 0,15 & NA & 0 & 1 \\
\hline 10 a 20000 & 0,26 & NA & 0 & 1 \\
\hline
\end{tabular}


TABELA 1. (Continued) Análise descritiva das variáveis associadas ao câncer de mama em municípios brasileiros, 1990 a 2010

\begin{tabular}{|c|c|c|c|c|}
\hline Variável $^{a}$ & Média & Desvio padrão & Mínimo & Máximo \\
\hline 50 a 100000 & 0,12 & NA & 0 & 1 \\
\hline 100 a 500000 & 0,10 & NA & 0 & 1 \\
\hline 500000 a 1000000 & 0,01 & NA & 0 & 1 \\
\hline Total & 1,00 & & & \\
\hline \multicolumn{5}{|l|}{ Tendência ${ }^{f}$} \\
\hline Brasil & NA & NA & 1 & 3 \\
\hline Norte & NA & NA & 0 & 3 \\
\hline Nordeste & NA & NA & 0 & 3 \\
\hline Sudeste & NA & NA & 0 & 3 \\
\hline
\end{tabular}

Fonte: DATASUS, IPEADATA e PNUD.

a GPS = gasto público per capita em saúde; IDHE = índice de desenvolvimento humano referente a educação; IDHL = índice de desenvolvimento humano referente a longevidade; IDHR = índice de desenvolvimento humano referente a renda; $\mathrm{MCM}$ = taxa de mortalidade por câncer de mama/100 000 habitantes; NES = número de estabelecimentos de saúde; NPS = número de profissionais de saúde com ensino superior.

b 0 valor de produção industrial per capita (em dólares para o ano de 2000) foi: US\$ 477,78 (1990); US\$ 618,93 (2000); US\$ 959,91 (2010). Média: US\$ 685,54.

c A conversão de reais em dólares americanos baseou-se na taxa de câmbio comercial (valor de venda), referente ao ano de 2000, disponibilizada pelo Banco Central do Brasil (cotação: $\mathrm{R} \$ / \mathrm{US} \$=1,83)$.

d 0 valor de gasto público per capita (em dólares para 0 ano de 2000) foi: US\$13,68 (1990); US\$ 43,13 (2000); US\$ 109,31 (2010). Média: US\$ 55,37.

${ }^{\text {e }}$ As médias das dummies geográficas e de porte municipal refletem a proporção de municípios pertencentes a cada categoria.

${ }^{\dagger} \mathrm{NA}=$ não se aplica.

TABELA 2. Modelos estimados para os municípios brasileiros contendo variáveis discretas associadas ao câncer de mama, 1990 a 2010

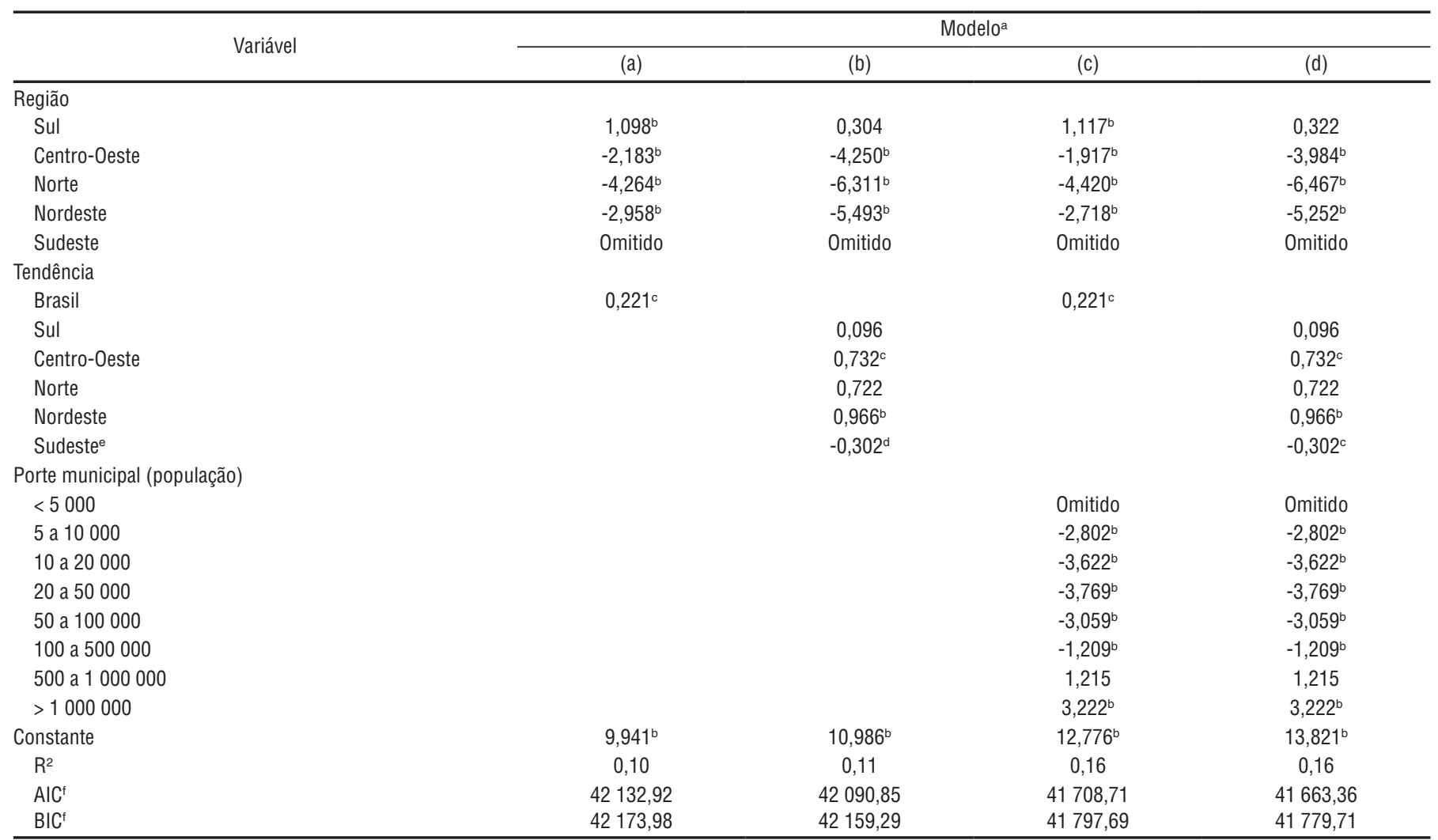

a Coeficientes de mortalidade por 100000 habitantes. Modelos estimados via pooled ordinary least squares (POLS). Modelos "a": inclui dummies de região + tendência e variável de tendência geral, válida para o Brasil (média dos municípios brasileiros). Modelos "b": inclui dummies de região + tendência + variáveis de tendência regional (uma para cada região).

Modelo "c": inclui dummies de região + variável de tendência geral + porte municipal. Modelo "d": inclui dummies de região + variável de tendência regional + porte municipal.

${ }^{b} P<0,001$ (teste t de Student).

${ }^{\circ} P<0,01$ (teste t de Student).

d $P<0,05$ (teste t de Student).

${ }^{\text {e }}$ Os municípios do Sudeste e aqueles com menos de 5000 habitantes são referência e, portanto, foram omitidos das estimações.

${ }^{\dagger}$ AIC = critério de informação de Akaike; BIC = critério de informação Bayesiano. 
TABELA 3. Modelos estimados para os municípios brasileiros contendo variáveis contínuas associadas ao câncer de mama, 1990 a 2010

\begin{tabular}{|c|c|c|c|}
\hline \multirow{2}{*}{ Variável $^{b}$} & \multicolumn{3}{|c|}{ Modelo ${ }^{\mathrm{a}}$} \\
\hline & (a) POLSC & (b) $\mathrm{RE}^{\mathrm{d}}$ & (c) $\mathrm{FE}^{\mathrm{e}}$ \\
\hline IDHR & $21,039^{\dagger}$ & $20,356^{\dagger}$ & 2,365 \\
\hline IDHL & 2,540 & 3,218 & $6,238^{g}$ \\
\hline IDHE & $-5,352^{\dagger}$ & $-5,332^{\dagger}$ & $-0,569$ \\
\hline Industrialização & 0,028 & 0,025 & $-0,015$ \\
\hline Fecundidade & $-0,468^{f}$ & $-0,382^{h}$ & $-0,018$ \\
\hline Mulheres jovens grávidas & $-0,196^{\dagger}$ & $-0,172^{f}$ & $-0,032$ \\
\hline População rural/urbana & $-0,010$ & $-0,004$ & 0,022 \\
\hline GPS & $-0,086$ & $-0,140^{g}$ & $-0,174^{9}$ \\
\hline NPS & 0,049 & 0,033 & $-0,029$ \\
\hline NES & 0,115 & 0,014 & 0,051 \\
\hline Constante & $-1,524$ & $-1,755$ & 4,148 \\
\hline $\mathrm{R}^{2}$ & 0,11 & 0,11 & 0,08 \\
\hline $\mathrm{AIC}^{\mathrm{i}}$ & 42032,87 & - & 37509,62 \\
\hline $\mathrm{BICi}$ & 42108,16 & - & 37584,90 \\
\hline
\end{tabular}

a Coeficientes de mortalidade por 100000 habitantes. Teste de Hausman (1978): (c) versus (b): $\chi^{2}=523,41$; probabilidade $>\chi^{2}=0,000$.

b GPS = gasto público per capita em saúde; IDHE = índice de desenvolvimento humano referente a educação; IDHL = índice de desenvolvimento humano referente a longevidade; IDHR = índice de desenvolvimento humano referente a renda; NES = número de estabelecimentos de saúde; NPS = número de profissionais de saúde com ensino superior.

C POLS = pooled ordinary least squares.

${ }^{d} \mathrm{RE}=$ random effects.

e $\mathrm{FE}=$ fixed effects.

f $P<0,001$ (t de Student).

${ }_{9} P<0,05$ (t de Student).

n $P<0,01$ (t de Student).

i $\mathrm{AIC}=$ critério de informação de Akaike; BIC =critério de informação Bayesiano.

Logo, a taxa de mortalidade desta região foi de 10,16 $(9,94+0,22)$ em 1990, 10,38 $(10,16+0,22)$ em 2000 e $10,60(10,38+$ $0,22)$ em 2010; a média foi igual a 10,38. Já a da região Sul foi de 11,26 (9,94+0,22 $+1,10$ ) (sendo 1,10 o valor arredondado do coeficiente associado a dummy da região Sul, de 1,098, como mostra a tabela 2) em 1990, 11,48 (11,26 + 0,22) em 2000 e $11,70(11,48+0,22)$ em 2010, com média de 11,48. As regiões Norte, Nordeste e Centro-Oeste apresentaram as menores taxas.

Segundo o modelo " $\mathrm{b}$ ", as regiões Nordeste $(0,97)$, Centro-Oeste $(0,73)$ e Norte $(0,72)$ foram as grandes responsáveis pela tendência de crescimento da mortalidade (tabela 2). Verificou-se ainda uma tendência negativa na região Sudeste $(-0,30)$ e uma estabilidade no Sul (onde o valor não foi significativo).

Segundo as dummies de porte municipal, modelo "c", as menores taxas de mortalidade são encontradas em municípios com 20 a 50000 habitantes. Já as maiores ocorreriam naqueles com mais de 1 milhão de habitantes $(9,22)$. Taxas elevadas também foram verificadas nos municípios com população entre 500000 e 1 milhão e naqueles onde a população não chegava a 5000 .

A inclusão conjunta das variáveis de porte municipal, região e tendência, modelos "c" e " $d$ ", afetou a magnitude, mas não a ordenação dos resultados. Portanto, presume-se que as análises anteriores permaneceram válidas (tabela 2).

O teste de Hausman (23) indicou que o modelo de efeitos fixos (tabela 3) foi preferível aos demais. Segundo esse modelo, a mortalidade por câncer de mama apresentou uma associação positiva/significativa com a longevidade e negativa/ significativa com o nível de gastos públicos em saúde.

Diferentemente dos resultados descritos na tabela 2, os modelos da tabela 4 indicaram uma tendência negativa/significativa associada à mortalidade por câncer de mama (modelos "a" $\mathrm{e}$ "b"). Os modelos " $c$ " $\mathrm{e}$ " $\mathrm{d}$ " revelaram que essa tendência negativa ocorreu em todas as regiões, sendo mais intensa no Sudeste (a cada decênio, a taxa diminuiria entre $-2,66$ e $-2,67 / 100000$ mulheres) e no Sul (cerca de $-2,29$ a $-2,30 / 100000$ mulheres).
A inclusão das variáveis explicativas contínuas não afetou a ordenação das dummies de região e porte municipal, mostrando que as análises anteriores permanecem válidas (tabela 4 ). A redução dos AIC e BIC apresentada na tabela 4, em relação aos resultados das tabelas 2 e 3, indicou uma melhora na especificação dos modelos. Apesar disso, o teste de Hausman (32) revelou que não foi possível eliminar completamente o problema causado pelos efeitos não observados. Logo, os resultados dos modelos de efeitos fixos foram os mais confiáveis.

\section{DISCUSSÃO}

Os resultados desta pesquisa indicam que a taxa de mortalidade por câncer de mama, padronizada por faixa etária e corrigida por causas mal definidas, cresceu no Brasil entre 1990 e 2010. Essa tendência difere da verificada em países desenvolvidos, onde a mortalidade é declinante $(3,14)$. É possível que fatores externos, como o crescimento da renda e da longevidade e a diminuição da taxa de fecundidade, tenham contribuído para esse crescimento.

As estimações indicaram que a mortalidade apresenta associação positiva/ significativa com a longevidade e negativa/significativa com o nível de gastos públicos em saúde. O resultado associado ao gasto público é coerente com o relatório da Organização Mundial da Saúde (OMS) (33), segundo o qual a redução de doenças não transmissíveis, como o câncer de mama, ainda requer um aumento significativo nos gastos com saúde em países em desenvolvimento. Quanto à longevidade, o achado é semelhante aos descritos na literatura $(1,14,16)$.

As dummies de região indicaram que a mortalidade é maior no Sul e Sudeste e menor no Norte, Nordeste e Centro-Oeste, respectivamente. Em parte, essa desigualdade se deve às peculiaridades regionais e já havia sido identificada anteriormente (13). Na realidade, os níveis elevados de renda e longevidade e a baixa taxa de fecundidade das regiões Sul e Sudeste, em relação às demais, já explicariam parte dessa diferença.

A consideração de algumas características socioeconômicas municipais mostrou que os coeficientes associados às dummies regionais se aproximaram de 
TABELA 4. Modelos estimados para os municípios brasileiros contendo variáveis discretas e contínuas associadas ao câncer de mama, 1990 a 2010

\begin{tabular}{|c|c|c|c|c|c|}
\hline \multirow{2}{*}{ Variáveis contínuas ${ }^{a}$} & \multicolumn{2}{|c|}{ Tendência geral } & \multicolumn{2}{|c|}{ Tendência regional } & \multirow{2}{*}{$\begin{array}{c}\text { Efeitos fixos } \\
(\mathrm{e}) \\
\mathrm{FE}^{\mathrm{d}}\end{array}$} \\
\hline & $\begin{array}{c}\text { (a) } \\
\text { POLS }^{b}\end{array}$ & $\begin{array}{l}\text { (b) } \\
\mathrm{RE}^{\mathrm{c}}\end{array}$ & $\begin{array}{c}\text { (c) } \\
\text { POLS }^{b}\end{array}$ & $\begin{array}{l}\text { (d) } \\
R^{c}\end{array}$ & \\
\hline IDHR & $9,063^{e}$ & $8,728^{\mathrm{e}}$ & $9,267^{e}$ & $8,856^{\mathrm{e}}$ & 2,365 \\
\hline IDHE & $5,865^{e}$ & $6,137^{e}$ & $6,754^{e}$ & $7,193^{\mathrm{e}}$ & $-0,569$ \\
\hline Industrialização & 0,018 & 0,015 & 0,021 & 0,018 & $-0,015$ \\
\hline População rural/população urbana & $-0,125^{\dagger}$ & $-0,108^{g}$ & $-0,120^{g}$ & $-0,102^{g}$ & 0,022 \\
\hline GPS & 0,061 & 0,029 & 0,094 & 0,065 & $-0,174^{9}$ \\
\hline NPS & $0,074^{\dagger}$ & $0,060^{9}$ & $0,088^{e}$ & $0,075^{\dagger}$ & $-0,029$ \\
\hline NES & $-0,542^{g}$ & $-0,415$ & $-0,603^{9}$ & $-0,487$ & 0,051 \\
\hline \multicolumn{6}{|l|}{ Região } \\
\hline Sul & $0,969^{e}$ & $0,935^{\mathrm{e}}$ & 0,253 & 0,218 & Omitido \\
\hline Sudeste & Omitido & Omitido & Omitido & Omitido & Omitido \\
\hline \multicolumn{6}{|l|}{ Porte municipal (população) } \\
\hline$<5000$ & Omitido & Omitido & Omitido & Omitido & Omitido \\
\hline 5 a 10000 & $-2,778^{e}$ & $-2,765^{e}$ & $-2,783^{e}$ & $-2,772^{\mathrm{e}}$ & Omitido \\
\hline 10 a 20000 & $-3,626^{e}$ & $-3,600^{e}$ & $-3,641^{e}$ & $-3,619 e$ & Omitido \\
\hline 20 a 50000 & $-4,283^{e}$ & $-4,241^{e}$ & $-4,309^{e}$ & $-4,270^{e}$ & Omitido \\
\hline 50 a 100000 & $-4,273^{e}$ & $-4,216^{e}$ & $-4,300^{e}$ & $-4,243^{e}$ & Omitido \\
\hline 100 a 500000 & $-3,062^{\mathrm{e}}$ & $-2,991^{e}$ & $-3,105^{e}$ & $-3,034^{e}$ & Omitido \\
\hline 500 a 1000000 & $-1,884^{f}$ & $-1,771^{9}$ & $-1,944^{\dagger}$ & $-1,826^{9}$ & Omitido \\
\hline$>1000000$ & $-0,553$ & $-0,419$ & $-0,628$ & $-0,487$ & Omitido \\
\hline \multicolumn{6}{|l|}{ Tendência } \\
\hline $\mathrm{R}^{2}$ & 0,21 & 0,21 & 0,21 & 0,21 & 0,08 \\
\hline$A I C^{i}$ & 41299,18 & - & 41268,08 & - & 37509,62 \\
\hline $\mathrm{BIC}^{\mathrm{i}}$ & 41456,59 & - & 41452,86 & - & 37584,90 \\
\hline
\end{tabular}

a Coeficientes de mortalidade por 100000 habitantes. GPS = gasto público per capita em saúde; IDHE = índice de desenvolvimento humano referente a educação; IDHL = índice de desenvolvimento humano referente a longevidade; IDHR = índice de desenvolvimento humano referente a renda; MCM = taxa de mortalidade por câncer de mama/100 000 habitantes; NES = número de estabelecimentos de saúde; NPS = número de profissionais de saúde com ensino superior.

b $\mathrm{POLS}=$ pooled ordinary least squares.

c $\mathrm{RE}=$ random effects.

${ }^{d} \mathrm{FE}=$ fixed effects. 0 estimador FE elimina automaticamente as variáveis que não apresentam variação entre os anos analisados (omitido).

e $P<0,001$ (teste t de Student).

${ }^{\dagger} P<0,01$ (teste $t$ de Student).

${ }^{9} P<0,05$ (teste t de Student).

h Os municípios do Sudeste e aqueles com menos de 5000 habitantes são referência e, portanto, foram omitidos das estimações.

' AIC e BIC são os critérios de informação de Akaike e Bayesiano, respectivamente.

zero. Portanto, as desigualdades socioeconômicas podem ter sido responsáveis por parte da diferença regional associada à mortalidade por câncer de mama. De fato, Sul e Sudeste permaneceram com taxas maiores que as demais regiões.
A análise de dummies de porte municipal mostrou as menores taxas de mortalidade nos municípios com 20 a 50000 habitantes. Já as maiores ocorreram tanto nos municípios com mais de 500000 habitantes quanto naqueles onde a população não chega a 5 000. No caso dos municípios pequenos, é possível que a falta de infraestrutura dificulte a detecção e o tratamento da doença. Por sua vez, nas localidades de grande porte, a elevada mortalidade poderia ser um reflexo do deslocamento de mulheres com câncer de mama para os grandes centros em 
busca de melhores tratamentos, além da urbanização e possíveis mudanças nos padrões reprodutivos. Gebrim e Quadros já haviam detectado esse gargalo nas grandes cidades do Brasil (20).

Inicialmente, a variável de tendência indicou um aumento significativo na taxa de mortalidade entre 1990 e 2010 no Brasil (tabela 2). Esse crescimento foi maior no Nordeste, Centro-Oeste e Norte, estável no Sul e negativo no Sudeste, o que é condizente com relatos anteriores $(34,35)$. É possível que o crescimento da renda e da longevidade e a redução na taxa de fecundidade verificados no Brasil no período considerado tenham impulsionado esse aumento na mortalidade. Quanto ao Sudeste, por ser a região mais rica, acredita-se que seus avanços na prevenção, detecção e tratamento do câncer de mama estejam permitindo uma redução na mortalidade, assim como em países mais desenvolvidos.

É interessante notar que, após considerar algumas variáveis socioeconômicas (isto é, renda, longevidade, fecundidade), a tendência associada à taxa de mortalidade se tornou negativa/significativa em todas as regiões e foi mais intensa no Sudeste e Sul e menor no Nordeste (tabela 4). A explicação para a inversão da tendência requer certa atenção. De modo geral, os resultados da tabela 2, sem considerar as variáveis contínuas (renda, longevidade, fecundidade), indicaram um crescimento da mortalidade (tendência positiva). Porém, após inclusão das variáveis contínuas, a tendência se tornou negativa, mostrando o que ocorreria com a mortalidade se tais fatores não tivessem variado no período. Entretanto, a renda e a longevidade cresceram entre 1990 e 2010, enquanto a fecundidade diminuiu.
Logo, como a renda, a longevidade, e a fecundidade apresentaram associações positiva, positiva e negativa em relação à mortalidade, respectivamente, é possível inferir que a variação desses fatores contribuiu, pelo menos em parte, para o crescimento verificado na mortalidade. Em outras palavras, caso fatores externos como renda, expectativa de vida e taxa de fecundidade, entre outros, não tivessem se alterado no período, as taxas de mortalidade poderiam ter sido reduzidas nas regiões brasileiras.

Apesar dos gastos públicos em saúde terem aumentado no período, ainda há necessidade de investimentos nessa área, principalmente em localidades de pequeno e grande porte (com menos de 5000 e mais de 500 000habitantes, respectivamente), onde a mortalidade se mostrou acima da média.

Cabe destacar que o uso de dados secundários agregados e a impossibilidade de inclusão de alguns fatores associados à mortalidade por câncer de mama constituem uma limitação deste trabalho. Além disso, a análise da mortalidade ao longo de 20 anos envolve outros aspectos de difícil incorporação nos modelos (por exemplo, avanços/alterações nos registros de óbito, nos diagnósticos e nos tratamentos do câncer de mama). Apesar disso, este trabalho permitiu identificar alguns fatores socioeconômicos que, possivelmente, contribuíram para o crescimento da mortalidade por câncer de mama no Brasil (por exemplo, crescimento da renda e da longevidade e redução da fecundidade). Foi ainda possível distinguir algumas características dos municípios com elevada mortalidade (porte pequeno/grande e pertencer às regiões Sul e Sudeste) e propor medidas para reduzir a mortalidade média no país (por exemplo, expandir os gastos públicos em saúde). Obviamente, devese considerar que boa parte dos municípios com baixas taxas de mortalidade são, na realidade, menos favorecidos (pobres) e, portanto, também precisarão de mais investimentos e políticas voltadas ao tratamento e detecção desta neoplasia à medida que suas rendas e taxas de longevidade aumentarem.

Como é possível que outras nações emergentes enfrentem transições socioeconômicas e demográficas semelhantes às vivenciadas pelo Brasil, é provável que também experimentem uma elevação significativa da mortalidade por câncer de mama. Portanto, é necessário que tais países estejam preparados. Com base nos resultados descritos aqui, é possível inferir que a mortalidade nesses países tenderá a se concentrar, primeiramente, nas grandes/pequenas cidades de suas regiões mais ricas.

Agradecimentos. Os autores agradecem à Coordenação de Aperfeiçoamento de Pessoal de Nível Superior (CAPES) pela bolsa de estudos (mestrado) concedida a MSAC durante a realização desta pesquisa. MTBT é bolsista de produtividade do Conselho Nacional de Desenvolvimento Científico e Tecnológico (CNPq).

Conflitos de interesse. Nada declarado pelos autores.

Declaração. As opiniões expressas no manuscrito são de responsabilidade exclusiva dos autores e não refletem necessariamente a opinião ou política da RPSP / PAJPH ou da Organização Pan-americana de Saúde (OPAS).

\section{REFERÊNCIAS}

1. Malta DC, Moura L, Souza MFM, Curado MP, Alencar AP, Coimbra R, et al. Tendência de mortalidade por câncer de mama no Brasil e em estados selecionados. Rev Min Enferm. 2008;12(2):21926.

2. Paulinelli RR, Freitas R, Júnior MPC, Souza A. A situação do câncer de mama em Goiás, no Brasil e no mundo: tendências atuais para a incidência e a mortalidade. Rev Bras Saude Mater Infant. 2003;3(1):17-24.

3. Lacey JV, Devessa SS, Brinton LA. Recent trends in breast cancer incidence and mortality. Environ Mol Mutagen. 2002;39(2-3): 82-8

4. Wunsch Filho V, Moncau JE. Mortalidade por câncer no Brasil 1980-1995: padrões regionais e tendências temporais. Rev Assoc Med Bras. 2002;48(3):250-7.

5. FerlayJ,Hery C,AutierP,Sankaranarayanan R. Global burden of breast cancer. Em: Li CI, ed. Breast cancer epidemiology. New York: Springer; 2010. Pp. 1-19.

6. Siegel R, Ma J, Zou Z, Jemal A. Cancer statistics, 2014. CA Cancer J Clin. 2014;64(1):9-29.

7. Silva GA, Gamarra CJ, Girianelli VR, Valente JG. Tendência da mortalidade por câncer nas capitais e interior do Brasil entre 1980 e 2006. Rev Saude Publica. 2011;45(6):1009-18.

8. Barbosa IR, De-Souza DL, Bernal MM, Costa CC. Cancer mortality in Brazil: temporal trends and predictions for the year 2030. Medicine (Baltimore). 2015;94(16):e746.
9. Martins CA, Guimarães RM, Silva RLPD, Ferreira APS, Gomes FL, Sampaio JRC, et al. Evolução da mortalidade por câncer de mama em mulheres jovens: desafios para uma política de atenção oncológica. Rev Bras Cancerol. 2013;59(3):341-9.

10. Girianelli VR, Gamarra CJ, Silva GA. Os grandes contrastes na mortalidade por câncer do colo uterino e de mama no Brasil. Rev Saude Publica. 2014;48(3):459-67.

11. Guerra MR, Gallo CVM, Mendonça GAS. Risco de câncer no Brasil: tendências e estudos epidemiológicos mais recentes. Rev Bras Cancerol. 2005;51(3):227-34.

12. Bray F, Jemal A, Grey N, Ferlay J, Forman D. Global cancer transitions according to 
the human development index (20082030): a population based study. Lancet Oncol.2012;13(8):790-801.

13. Silva GA, Teixeira MTB, Aquino SML, Tomazelli JG, Silva IS. Acesso à detecção precoce do câncer de mama no Sistema Único de Saúde: uma análise a partir dos dados do Sistema de Informações em Saúde. Cad Saude Publica. 2014;30(7):1537-50.

14. Althuis MD, Dozier JM, Anderson WF, Devesa SS, Brinton LA. Global trends in breast cancer incidence and mortality 19731997. Int J Epidemiol. 2005;34(2):405-12.

15. Boing AF, Vargas SAL, Boing AC. A carga de neoplasias no Brasil: mortalidade e morbidade hospitalar entre 2002-2004. Rev Assoc Med Bras. 2007;53(4):317-22.

16. Di-Pietro PF, Medeiros NI, Vieira FG, Fausto MA, Belló-Klein A. Breast cancer in southern Brazil: association with past dietary intake. Nutr Hosp. 2007;22(5):565-72.

17. Hussain SK, Lenner P, Sundquist J, Hemminki K. Influence of education level on cancer survival in Sweden. Ann Oncol. 2008;19(1):156-62.

18. Thuler LC. Considerações sobre a prevenção do câncer de mama feminino. Rev Bras Cancerol. 2003;49(4):227-38.

19. Rezende MCR, Koch HA, Figueiredo JA, Thuler LCS. Causas do retardo na confirmação diagnóstica de lesões mamárias em mulheres atendidas em um centro de referência do Sistema Único de Saúde no Rio de Janeiro. Rev Bras Ginecol Obstet. 2009;31(2):75-81.

20. Gebrim LH, Quadros LGA. Rastreamento do câncer de mama no Brasil. Rev Bras Ginecol Obstet. 2006;28(6):319-23.

21. Faria MAM, Almeida JWR, Zanetta DMT. Mortalidade por câncer na região urbano-industrial da Baixada Santista, SP
(Brasil). Rev Saude Publica. 1999;33(3): 255-61.

22. Departamento de Informática do SUS(DATASUS). Informações de saúde (TABNET).Disponível em: http://www2. datasus.gov.br/DATASUS/index.php/index.php?area=02 Acessado em 10 de fevereiro de 2016.

23. França E, Teixeira R, Ishitani L, Duncan BB, Cortez-Escalante JJ, Neto OLM, et al. Causas mal definidas de óbito no Brasil: método de redistribuição baseado na investigação do óbito. Rev Saude Publica. 2014;48(4):671-81.

24. Mathers CD, Bernard C, Iburg KM, Inoue M, Fat DM, Shibuya K, et al. Global burden of disease in 2002: data sources, methods and results. Genebra: World Health Organization; 2003. Disponível em: http:/ / www.who.int/healthinfo/paper54.pdf Acessado em 5 de setembro de 2017

25. Instituto de Pesquisa Econômica Aplicada. IPEADATA. Regional. Disponível em: www.ipeadata.gov.br/ Acessado em 10 de fevereiro de 2016.

26. Bray F, Ferlay J. Age standardization. Em: Forman D, Bray F, Brewster DH, GombeMbalawa C, Kohler B, Pineros M et al., ed. Cancer incidence in five continents. Lyon: International Agency for Research on Cancer;2014. Volume X. Pp. 112-115. IARC Scientific Publication No. 164. Disponível em: https://www.iarc.fr/en/publications/ pdfs-online/epi/sp164/CI5volX_Full.pdf Acessado em 5 de setembro de 2017.

27. Programa das Nações Unidas para o Desenvolvimento(PNUD). Atlas do desenvolvimento humano dos municípios. Disponível em: http://www.br.undp.org/ content/brazil/pt/home/idh0/atlas-dod e s envolvimento-humano / atlas-dos-municipios.html Acessado em 10 de fevereiro de 2016.

28. Cintra JRD, Teixeira MTB, Diniz RW, Junior HG, Florentino TM, Freitas GF, et al. Perfil imuno-histoquímico e variáveis clínico-patológicas no câncer de mama. Rev Assoc Med Bras.2012;58(2):178-87.

29. Greene WH. Econometric analysis. 5th ed. New Jersey: Prentice Hall; 2002.

30. Hsiao C. Analysis of panel data. $2^{a}$ ed. Cambridge: Cambridge University Press; 2003.

31. Wooldridge JM. Econometric analysis of cross section and panel data. London: MIT Press; 2002.

32. Hausman JA. Specification tests in econometrics. Econometrica.1978;46(6):1251-71.

33. Organização Mundial da Saúde (OMS). Relatório mundial da saúde. Financiamento dos sistemas de saúde: o caminho para a cobertura universal. Disponível em: http:/ / www.who.int/eportuguese/publications/WHR2010.pdf Acessado em 5 de setembro de 2017.

34. Kluthcovsky ACGC, Faria TNP, Carneiro FH, Strona R. Female breast cancer mortality in Brazil and its regions. Rev Assoc Med Bras. 2014;60(4):387-93.

35. Freitas-Junior R, Gonzaga CMR, Freitas NMA, Martins E, Dardes RCM. Disparities in female breast cancer mortality rates in Brazil between 1980 and 2009. Clinics (Sao Paulo). 2012;67(7):731-7.

Manuscrito recebido em 22 de abril de 2017. Aceito em versão revisada em 9 de agosto de 2017.

ABSTRACT Objective. To analyze breast cancer mortality trends in Brazilian municipalities and assess the influence of socioeconomic and demographic factors on mortality rates.

Methods. Age-adjusted mortality rates were calculated for the periods centered in 1990, 2000, and 2010 and corrected for ill-defined causes of death. After that, panel data regression models were developed for analysis of the association between the factors of interest and the mortality rate from breast cancer in Brazilian municipalities.

Breast cancer mortality in Brazilian municipalities and associated factors

Results. A growing trend was detected in breast cancer mortality in Brazil. However, the models showed that the mortality could have decreased (negative trend), especially in the Southeast and South regions, if some associated factors (such as income, education, longevity, fertility rate, health spending, and infrastructure, among others) had remained constant during the study period. Breast cancer mortality was positively/ significantly associated with longevity and negatively/significantly associated with public health spending. Mortality was higher in the South and Southeast, in municipalities with more than 500000 inhabitants and in those with population below 5000.

Conclusions. The growth in per capita income, the increase in life expectancy, and the decrease in fertility rates may be associated with high breast cancer mortality and a trend towards increased mortality from this cancer in Brazilian municipalities. 
RESUMEN

\section{Mortalidad por cáncer de} mama en municipios brasileños y factores asociados

Palabras claves
Objetivo. Analizar las tendencias de la mortalidad por cáncer de mama en municipios brasileños y evaluar la influencia de factores socioeconómicos y demográficos sobre las tasas de mortalidad.

Métodos. Se calcularon las tasas de mortalidad, ajustadas por grupo etario y corregidas por causas mal definidas, para los años 1990, 2000 y 2010. Posteriormente, se calcularon modelos de regresión, a partir de datos de panel, que permitieron verificar el grado de asociación entre distintos factores de interés y la tasa de mortalidad por esta enfermedad. Resultados. Se verificó que había una tendencia al aumento de la mortalidad en el país. Sin embargo, los modelos indicaron que la mortalidad podría haber disminuido (tendencia negativa), principalmente en el sudeste y el sur, si algunos factores asociados (por ejemplo, nivel de ingresos, educación, longevidad, tasa de fecundidad, gastos en salud, infraestructura, entre otros) hubieran permanecido constantes durante el período en estudio. Se observó que la mortalidad por cáncer de mama presentó una asociación positiva y significativa con la longevidad, y negativa y significativa con el nivel del gasto público en salud. La mortalidad fue mayor en las zonas sur y sudeste, en los municipios con más de 500000 habitantes y en aquellos cuya población es inferior a 5000.

Conclusiones. El aumento de los ingresos per cápita, el incremento de la esperanza de vida y la disminución de la tasa de fecundidad pueden estar asociados a tasas altas de mortalidad por cáncer de mama y a una tendencia al aumento de dicha mortalidad en los municipios brasileños.

Neoplasias de la mama; mortalidad; epidemiología; métodos y procedimientos estadísticos; Brasil. 